\title{
A 10-17 DOF Sensory Gloves with Harvesting Capability for Smart Healthcare
}

\author{
V. Stornelli, A. Leoni, G. Ferri, V. Errico, A. Pallotti, G. Orengo, and G. Saggio
}

\begin{abstract}
We present a 10-17 Degrees of Freedom (DoF) sensory gloves for Smart Healthcare implementing an energy harvesting architecture, aimed at enhancing the battery lasting when powering the electronics of the two different types of gloves, used to sense fingers movements. In particular, we realized a comparison in terms of measurement repeatability and reliability, as well as power consumption and battery lasting, between two sensory gloves implemented by means of different technologies. The first is a 3D printed glove with $10 \mathrm{DoF}$, featuring low-cost, loweffort fabrication and low-power consumption. The second is a classical Lycra ${ }^{\circledR}$ glove with 14 DoF suitable for a more detailed assessment of the hand postures, featuring a relatively higher cost and power consumption. An electronic circuitry was designed to gather and elaborate data from both types of sensory gloves, equipped with flex sensors, differing for number of inputs only. Both gloves allow the control of hand virtual limbs or mechanical arts in surgical, military, space and civil applications. The proposed gloves were already individually evaluated in terms of repeatability, reproducibility and reliability, but in this work their performances are compared also in terms of power consumption, because a particular effort was devoted in this case to increase battery lasting for both systems, developing an Energy Harvesting (EH) system with the electronics relaying on Radio Frequency, Piezoelectric and Thermoelectric harvesters, and applying it to the gloves for the first time. The harvesting part was built and tested as a prototype discrete element board, that is interfaced with an external microcontroller and a radiofrequency transmitter board. Measurement results demonstrated a meaningful improvement in battery operation time up to $25 \%$, considering different operating scenarios, for both glove systems, which exhibited not very different power consumption and therefore battery duration, in spite of different DoF measuring capabilities.
\end{abstract}

Index Terms-Sensory glove, Energy harvesting, power management.

\section{INTRODUCTION}

$\mathrm{T}$ he energy recovery from ambient power sources, the socalled energy harvesting, is an interesting way to capture and store energy for powering electrical devices, as those used in wearable electronics and remote wireless sensor network

Manuscript received January 15, 2019; revised March 5, 2019. Date of publication April 15, 2019. Date of current version June 3, 2019.

V. Stornelli, A. Leoni, G. Ferri are with the Dept.of Industrial and Information Engineering and Economics P.le Pontieri 1, 67100, Monteluco di Roio L'Aquila, Italy (emails: vincenzo.stornelli@univaq.it, alfiero.leoni@graduate.univaq.it, Giuseppe.ferri@univaq.it). V. Errico, A Pallotti, G. Orengo, G. Saggio are with the Dept.of Electronic Engineering via del Politecnico 1, 00133, Roma, Italy (emails: vito.errico@uniroma2.it, antonio.pallotti@uniroma2.it, orengo@uniroma2.it,saggio@uniroma2.it).

Digital Object Identifier (DOI): 10.24138/jcomss.v15i2.693
(WSN) [1-9]. Once the energy is recovered, the next step is to collect it in appropriate storage systems. With this aim, batteries are the most important storage block for autonomous devices, but they have a limited time duration that can be enlarged by the harvested free energy. Several efforts have been devoted to developing power harvesting techniques used to scavenge power either from the environment or from the human body [10]. Among the Energy Harvesting (EH) applications, in the last years a lot of interest has been devoted on sensory glove [11-13], both for healthcare and ludic application, in order to guarantee a long time operation $[14,15]$. The battery life time of the sensory glove can be harvested by means of different energy harvesters, operating all together such as electromagnetic, solar, thermal, acoustic, vibrational, etc.

In order to guarantee the glove comfort and portability we considered the development of an unwired, size- and weightreduced system, which exploits multi-sources energy harvesting techniques in order to extend the battery lasting.

In this work we propose a couple of 10-17 degrees of freedom (DoF) sensory gloves for Smart Healthcare, implementing a multisource energy harvesting architecture, aimed at enhancing the battery lasting when powering the electronics of the two different types of gloves, used to sense fingers movements. The two gloves have different characteristic: the former is a 3D printed glove with 10 flex sensors on finger joints for $10 \mathrm{DoF}$ hand motion assessment, the latter is a Lycra glove with 14 flex sensors on finger joints for 14 DoF hand motion assessment, requiring more power consumption. The performances of the presented gloves, which were already individually evaluated in terms of repeatability, reproducibility and reliability [16,17], are now compared also in terms of power consumption, testing them on six healthy subjects by mean of the Wise test [15]. The proposed multiharvester system integrates Thermo-Electric Generators (TEGs) (that can be applied on the human forearm), stacked piezoelectric (PZT) disks, placed in the heel of a shoe, so to scavenge energy from the pressure generated by feet during walking or a running session, and RF dual band circuitry to capture energy from the available surrounding RF power. The harvesting part was built and tested as a prototype discrete element board, that is interfaced with an external microcontroller and a radiofrequency transmitter board. Measurement results demonstrated a meaningful improvement 
in battery operation time up to $25 \%$, considering different operating scenarios.

\section{The Proposed LONG-LiFE B ATTERY TIME GLOVES}

For human body hands are fundamental to interact with the surroundings and to communicate too. A sensory glove allows to dynamically control the fingers and hand movements [14] in order to use the acquired data for several applications as among others: gaming, training or rehabilitation, evaluation of surgical gesture [12-20]. Generally, a sensory glove is made by an elastic glove with accelerometer or flex sensors embedded [13], generating electric signals according to fingers movements [14]. The hand movement capabilities are generally represented by 27 DoFs [20,21], so to take into account both flexion/extension and adduction/abduction of the finger joints as well as rotation/bending of the wrist. However, for usual applications, we can limit to a sub-set of DoFs. In particular, in the following we consider two gloves with different capabilities, a simple 3D printed glove with 10 DoFs, and a more sophisticated glove with a combination of 14 flex sensors and one inertial measurement unit (IMU), which are compared in terms of repeatability, reproducibility and reliability of the measures as well as power consumption.

\section{A. From 10 to 14 DoF Gloves}

A 3D printed glove with 10 DoF was built as a single fabric without seams or welding or use of glues, according to the anatomy of the hand [17].

The glove design includes the housings for the flex sensors [14,26] (Flexpoint Sensor Systems Inc., South Draper UT, USA) on distal interphalangeal (DIP), proximal interphalangeal (PIP), and metacarpophalangeal (MCP) finger joints, which are pockets or two foils within which the sensor can slide during joint bending. As a future improvement, the sensor can be integrated into the glove during the printing process, in order to guarantee each sensor to be correctly fixed in its location.

Figure 1 shows a photo of the realized glove from the CAD design, and the wiring of the flex sensors, taken from a Lycra glove and applied to the printed glove without any modification.

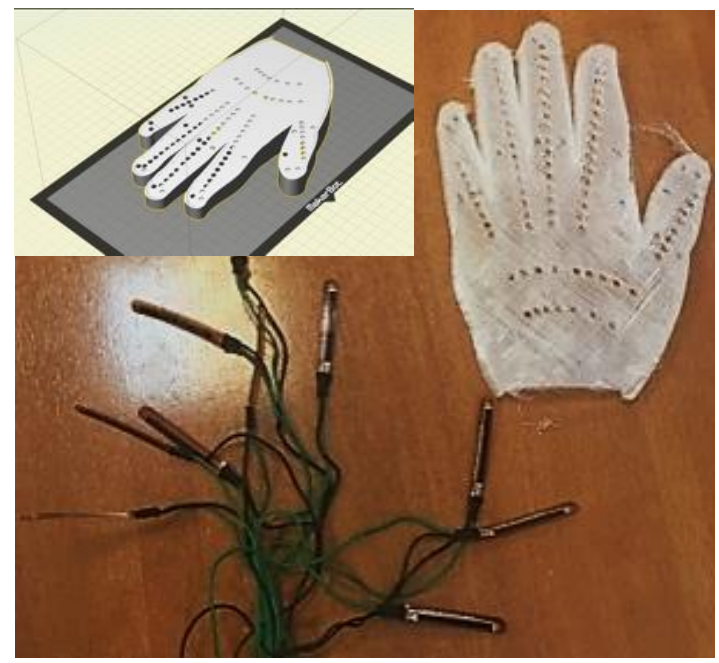

Fig. 1. Flex sensors' wiring (left down) and 3D printed prototype (right up) of the CAD designed glove (left up) [17]
In order to measure the hand gestures, we developed also a sensory glove equipped with 14 flex sensors (Flexpoint Sensor Systems, Inc., Draper, UT) and a 3-axis accelerometer (ADXL335, by Analog Devices, Inc., Norwood, MA) able to measure the flex/ extension capabilities of the finger joints of a human hand, plus the wrist movements. Flex sensors were positioned on distal interphalangeal, proximal interphalangeal, and metacarpo- phalangeal finger joints, except for the thumb that has a proximal IP (PIP) sensor and distal IP sensor (DIP), as shown in Fig. 2. The accelerometer was positioned on back side of the hand. In total 17 signals were collected by means of a custom-made prototype board connected to a computer, so to take into account of both flexion/extension of the joints of the fingers and rotation/bending of the wrist. The complete wearable system can be seen in Fig. 3.

The selected resistive sensors are stable, low cost, with a thickness less than 5 mils, flexible, almost linear [21,26] Different sensor sizes can be also selected depending on the application: sensors of 1, 2,3-inches are available for the finger joints DIP, PIP, MCP, respectively. The influence of fabric composition on sliding and flexion of the sensor has been studied. The glove is made of $88 \%$ polyester and $12 \%$ Elastane, because it provides greater comfort for movement. For the present study, only one medium right-hand gloves were developed. because could be best worn by the largest number of participants. The used sensory glove covers all the hand, but it can be modified to let the palmar surface and the fingertips free to maintain the tactile sensitivity. The wiring connection of the sensors to the electronic board is flexible and with a diameter of $1.5 \mathrm{~mm}$ and a weight less than $1 \mathrm{~g} / \mathrm{m}$. The sensory glove has a $50 \mathrm{~g}$ weight.

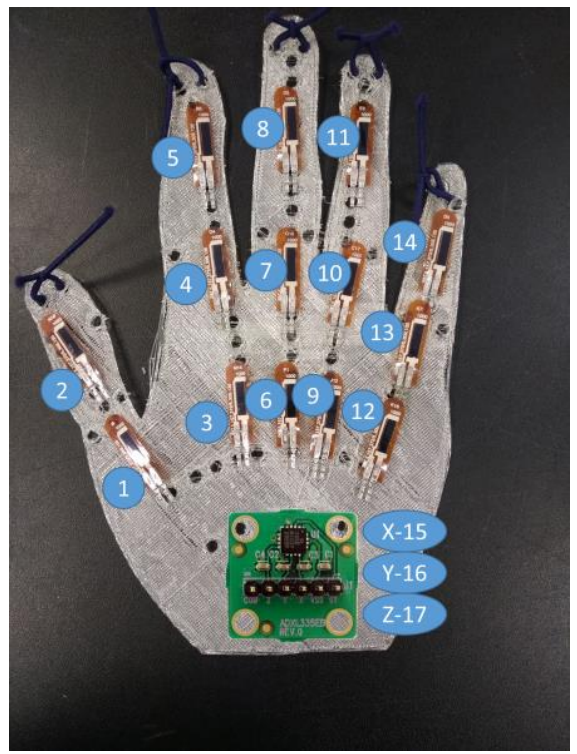

Fig. 2. Flex sensor position on the fin*ger joints of the 17 DoF glove, and IMU position on the back side of the hand.

\section{B. Data Acquisition and Transmission Board}

The Arduino Leonardo electronic board allows the management of 14 analog inputs for the bend sensor signals, 2 digital pin for Bluetooth serial communication, and provides ground and voltage reference for operation of the bend sensors. 


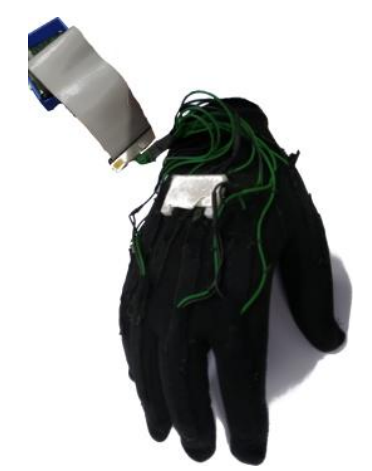

Fig. 3. The Lycra glove system: the glove with 14 flex sensors on the DIP, IP and MCP joint and the IMU device, and the acquisition and transmission box

The transduction takes place via a voltage divider between 0 and $5 \mathrm{~V}$ with a fixed $18 \mathrm{k} \Omega$ resistance, selected because it is the geometric mean between the maximum and minimum sensor resistance, to provide the widest voltage range [25]. The sensor readout circuit is followed by a 10-bit ADC module with a sampling frequency set to $1 \mathrm{kHz}$ and the transmission frequency set to $50 \mathrm{~Hz}$, to handle up to 20 multiplexed channels. Once the data are acquired, they are redirected to an RN42 Bluetooth module and sequentially to the receiving antenna connected to a PC. The current draw is about $100 \mathrm{~mA}$ for the $3 \mathrm{D}$ printed glove with 10 flex sensor, and $110 \mathrm{~mA}$ for the Lycra glove with 14 flex sensors and the IMU device, but is largely due to the acquisition and transmission board, since the bending sensor circuit has resistances of hundreds of Kohms and the IMU unit only absorbs $350 \mu \mathrm{A}$.

\section{Energy Harvesting System Architecture}

Evidently, practical reasons impose a wireless transmission system of the sensory gloves. The glove data transmission block scheme trough wireless connectivity is represented in Fig. 4. The system is composed by a RF commercial transmitting block driven by a microcontroller and a remote receiving block connected to a data server. In order to guarantee a system battery long life time, the overall system includes a multiharvester block, so as to scavenge free available energy directly from the surrounding environment, as demonstrated in [29-33]. The real case scenario is shown in Fig. 5. The transmitting and receiving blocks have been implemented with market components.

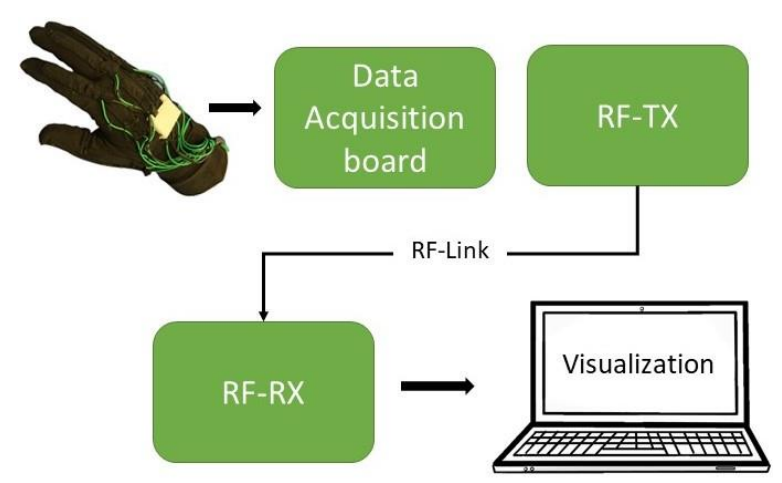

Fig. 4. Data glove acquisition and visualization process.

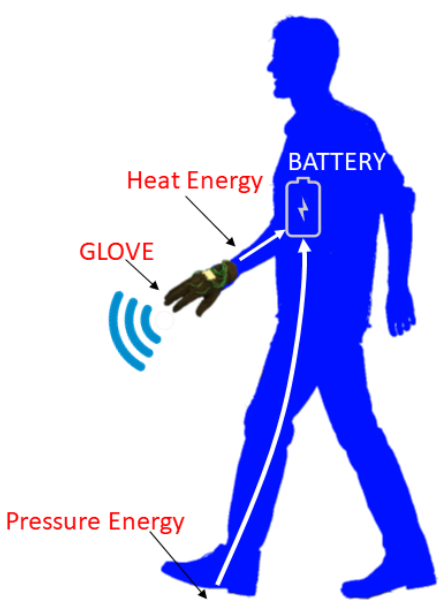

Fig. 5. The complete system block scheme

The harvesting block architecture is shown in Fig. 6. Three different harvesters terminated with a Schottky diode so to avoid reverse current flow operate in parallel. Details of the hardware implementation have been already reported in [33]. The energy coming from the human heat is collected by means of a set of 6 standard $2 \mathrm{~cm} \times 2 \mathrm{~cm}$ Peltier cells. The cells were arranged in series connection as depicted in Fig. $7 \mathrm{a}$, in order to achieve a higher output voltage. The cell surface is $55 \%$ smaller than the one employed in previous works. This reduces the harvested power per cell, but increases the power per area, since a smaller surface helps to achieve a better adhesion with the human forearm skin. Concerning the piezo harvesting branch (Fig. 7b), we adopted a parallel configuration of multiple stacked piezoelectric disks. Finally, for the RF harvester, a multichannel harvester operating at $936 \mathrm{MHz}$ and $2.4 \mathrm{GHz}$ with a dual band commercial antenna (Fig. 7c) and power selected incoming path as in [8].

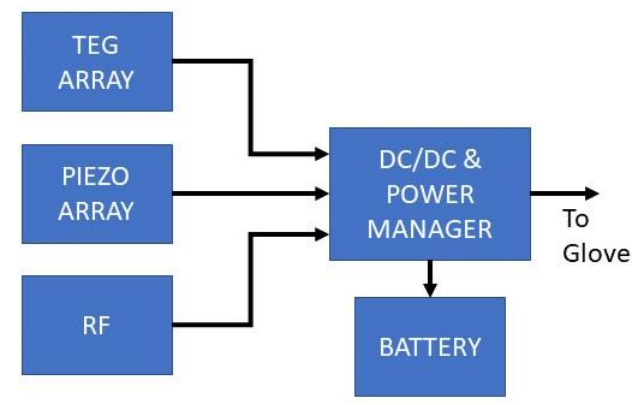

Fig. 6. Blocks representing the multi-harvesting system. It includes a thermal, pressure and RF energy harvesting paths, connected to a power management block.

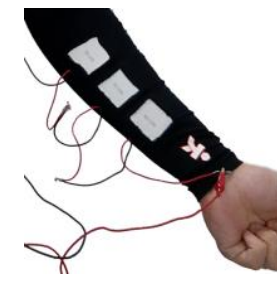

a)

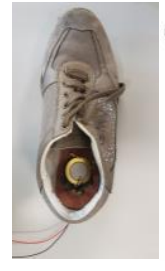

b)

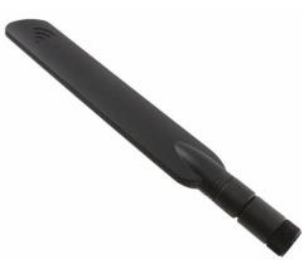

c)
Fig. 7. a) TEG Harvester; b) PZT Harvester; c) RF Antenna 


\section{MEASUREMENTS METHOD}

To compare the glove performances, the Wise test was conducted on the developed gloves $[16,19]$. Six healthy subjects were involved, 4 males and 2 females, all right-handed, 40 years mean age with $\mathrm{SD}= \pm 20$ years. The measurement protocol was approved by the local ethics committee. The glove was worn by the right hand and the electronic board was attached to the forearm. The measuring setup consisted of 4 areas: the area where to place the open hand at rest, the large mold area, the small mold area and the closed hand area. The hand postures during the Wise test are shown in Fig. 8.

The large mold is a 3D printed cylinder of $63 \mathrm{~mm}$ diameter, and the small mold of $53 \mathrm{~mm}$. These two sizes have been selected because they are between open and closed hand: the mean values, measured with a $3 \mathrm{D}$ printed goniometer with 1degree sensitivity, are 30 degrees for MCP and PIP with large mold, whereas they are 45 degrees for MCP and PIP for small mold.

The Wise test provides a mold gripping task A (Fig. 8 postures 2, 3 or 4 ) and a resting hand task $\mathrm{C}$ (posture 1). At this stage, the unit was never removed to study the repeatability of the sensors. A second stage with B in the same position of A and $\mathrm{D}$ in the same position of $\mathrm{C}$, the sensor unit was removed and worn again to study the reproducibility of the sensors. A time slot of 10 seconds for recording each position was selected from which the 6 central seconds were extracted for the analysis. The test was repeated for 10 iterations, then for 10 blocks (with a break of at least 3 minutes between two consecutive blocks for doffing and donning the glove). A first code deals with the organization of data, whereas a second code is used to calculate the Range and standard deviation (SD) values, intra-correlation coefficients (ICCs), and correlation between Range and SD for all subjects. The bending angles of the hand joints were measured without wearing the data gloves, using a manual goniometer with 1-degree resolution; the obtained values were used in calibration for mapping the digital values into angular values.

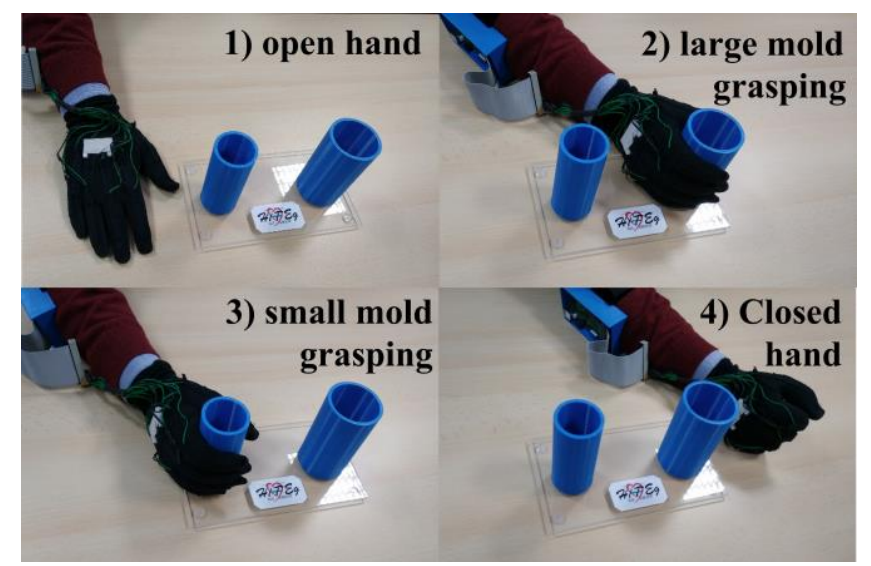

Fig. 8. Hand postures during Wise test: 1) open hand, 2) large mold grasping 3) small mold grasping, 4) hand completely closed.

\section{MEAsuREMENT Results}

\section{A. Glove performance comparison}

Measurements performed by the 3D printed glove and the Lycra glove were saved in the Matlab workspace as two 5- dimensional matrices indexed by the trial number (10), block number (10), joint or sensor number (10), position number (4) and subject (6). The Range and SD values were computed among the trials, and the mean across blocks, joints, positions and subjects is presented in Table I to evaluate repeatability (task A and C) and reproducibility (task B and D) in comparison with the results obtained by Simone [19], who applied only 5 flex sensors on the MCP joints, but did not considered the thumb, and Gentner [15], who applied 10 flex sensors on MCP and PIP joints like our 3D printed glove.

TABLE I

MEASUREMENT RESULTS OF THE 3D PRINTED AND LYCRA GLOVE IN TERMS OF REPEATABILITY (TASK A-C) AND REPRODUCIBILITY (TASK B-D) COMPARED WITH OTHER GLOVES FROM LITERATURE.

\begin{tabular}{|c|c|c|c|c|c|c|c|c|c|c|c|}
\hline \multirow{2}{*}{ fleve } & \multirow{2}{*}{ oF } & \multicolumn{2}{|c|}{ Task A } & \multicolumn{2}{|c|}{ Task B } & \multicolumn{2}{|c|}{ Task C } & \multicolumn{2}{|c|}{ Task D } & \multicolumn{2}{|c|}{ Mean } \\
\hline & & Range & SD & Range & SD & Range & SD & Range & SD & Range & SD \\
\hline & & & & & & & & & & & 2.2 \\
\hline & & & 2. & & & 3.47 & 1.11 & 4.4 & 1.44 & 5.36 & 1.71 \\
\hline entn & 10 & 6.09 & 1.94 & 7.16 & 2.26 & 2.61 & 0.86 & 3.98 & 1.28 & 4.96 & 1.59 \\
\hline Simone & 5 & 5.22 & 1.61 & & & 1 & 0.5 & & & 3.36 & 1.05 \\
\hline
\end{tabular}

The reliability between measures in each task was assessed by intraclass correlation coefficients (ICCs) [16]. The ICC calculation was based on within-subject variance, which reflects measurement errors. If within-subject variance is low, the ICC approaches 1 and the measurements are considered as reliable. Conversely, if the ICC approaches 0, a large fraction of variance is explained by measurement errors (indicating a low reliability). The mean out of 20 ICC calculations for each joint was used as a measure of joint sensor reliability. Thus, for each joint, four ICC values (one for each task) existed. The mean ICC for each joint across tasks served as a measure of reliability for a specific joint.

ICC values are reported in Table II, which are comparable to gloves evaluated by Gentner [15] and Simone [19]. Consequently, the repeatability and reliability of the 3D printed, and the Lycra gloves are similar to other evaluated gloves and also lies within the measurement reliability of manual goniometry (0.7) [15].

\section{TABLE II}

COMPARISON OF RELIABILITY, EXPRESSED AS INTRACLASS CORRELATION COEFFICIENTS (ICCS) RESULTING FROM THE WISE TEST, BETWEEN THE 3D PRINTED GLOVE WITH 10 DOF, THE LYCRA GLOVE WITH 17 DOF AND OTHER GLOVES FROM THE LITERATURE

\begin{tabular}{ccccc}
\hline \hline \multirow{2}{*}{ Glove } & \multirow{2}{*}{ DoF } & \multicolumn{3}{c}{ ICC } \\
\cline { 3 - 5 } & & Min & Max & Mean \\
\hline Printed & 10 & 0.69 & 0.83 & 0.73 \\
Lycra & 17 & 0.71 & 0.90 & 0.75 \\
Gentner & 14 & 0.87 & 0.98 & 0.93 \\
Simone & 4 & 0.79 & 1 & 0.95 \\
\hline \hline
\end{tabular}

\section{B. Energy Harvesting Results}

With respect to previous published papers $[33,34]$ the multi harvester block has been optimized in terms of working efficiency. Table III summarizes the achieved results in terms of conversion efficiency and harvested power.

Regarding the TEG harvested power, considering an average ambient temperature of $22^{\circ} \mathrm{C}$, a conversion efficiency peak of 
about $60 \%$ was achieved for an equivalent $10 \mathrm{k} \Omega$ load. For the PZT harvester measuring procedure, we employed an equivalent resistive load and a $75 \mathrm{~kg}$ human walking at $1 \mathrm{~Hz}$ speed on average. Measures showed in Table III demonstrate how the conversion efficiency peak, thus the maximum harvested power was achieved at lower loads, around $1 \mathrm{k} \Omega$. To conclude, Figure 9 shows the overall multichannel RF path conversion efficiency, which is the combination of both lowpower and medium-power harvesting channels, covering a -20 to $5 \mathrm{dBm}$ window of incoming power. The combination of the two RF harvesting channels allows to obtain a good conversion efficiency for an extended input power range, as demonstrated in [33-38].

The capabilities of the presented harvesting system where tested by implementing a low-power acquisition and transmission board as a benchmark platform. The system involves a microcontroller ARM Cortex M0 (by Microchip) and a Si4463 transceiver (by Silicon Labs) whose output transmission power was set to $0 \mathrm{dBm}$. The overall current is about $4 \mathrm{~mA}$ in idle mode, and as-low-as $10 \mathrm{~mA}$ in transmission mode. According to [9], by exploiting a power saving transmission algorithm, it is possible to achieve a power consumption of $13 \mathrm{~mA}$ on average, regardless of the chosen transmission baud rate (19200 or 38400 ), which in combination with the average harvested power, can extend the battery lasting of about $25 \%$.

TABLE III

SUMMARY OF THE ACHIEVED EFFICIENCY AND HARVESTED POWER FOR THE TEG ARRAY AND PIEZOELECTRIC HARVESTER, RESPECTIVELY, WITH RESPECT TO THE EQUIVALENT OUTPUT LOAD.

\begin{tabular}{ccc}
\hline \hline $\begin{array}{c}\text { Equivalent Load } \\
{[\mathrm{k} \Omega]}\end{array}$ & $\begin{array}{c}\text { TEG Conversion } \\
\text { Efficiency }[\%]\end{array}$ & $\begin{array}{c}\text { Piezo Harvested } \\
\text { Power }[\mathrm{mW}]\end{array}$ \\
\hline 1 & 32 & 0.8 \\
2 & 37 & 0.55 \\
5 & 47 & 0.3 \\
10 & 58 & 0.26 \\
20 & 49 & 0.22 \\
50 & 29 & 0.2 \\
100 & 38 & 0.3 \\
\hline \hline
\end{tabular}

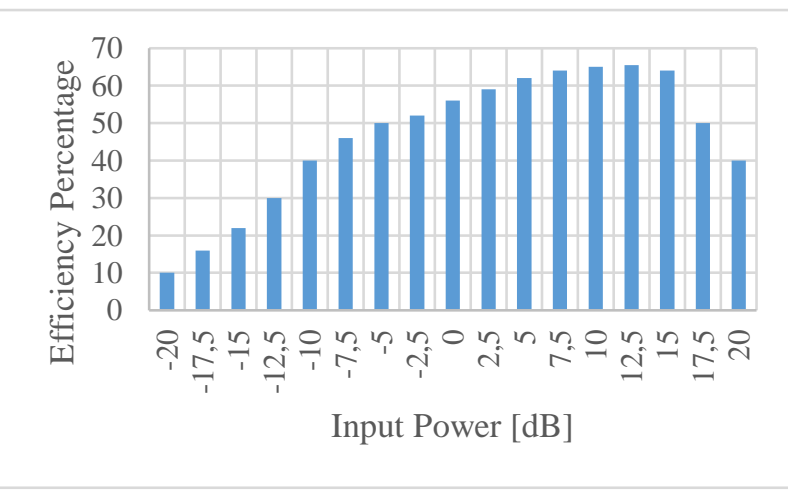

Fig. 9. RF path conversion efficiency [8].

\section{CONCLUSION}

We have presented a 10-17 degrees of freedom sensory gloves for Smart Healthcare, realized by means of different technologies, implementing an energy harvesting architecture.
The harvesting circuitry, able to scavenge energy from RF, Thermo-Electric Generators and piezoelectric disks, was tested, demonstrating the feasibility towards low supply-powered IC applications.

The Wise test with six healthy subjects was conducted on the selected gloves to make a comparison in terms of measurement repeatability, reproducibility and reliability, as well as power consumption and battery lasting. It can be shown that the repeatability, reproducibility and reliability of the $3 \mathrm{D}$ printed and the Lycra gloves with energy harvesting capability are similar to other evaluated gloves and also lies within the measurement reliability of manual goniometry, but the battery duration is increased by $25 \%$.

\section{REFERENCES}

[1] K. Al Agha, M.-H. Bertin, T. Dang, A. Guitton, P. Minet, T. Val, and J.B. Viollet, "Which Wireless Technology for Industrial Wireless Sensor Networks? The Development of OCARI Technology," IEEE Transactions on Industrial Electronics, vol. 56, no. 10, pp. 4266-4278, Oct. 2009. Doi: 10.1109/tie.2009.2027253

[2] V. C. Gungor and G. P. Hancke, "Industrial Wireless Sensor Networks: Challenges, Design Principles, and Technical Approaches," IEEE Transactions on Industrial Electronics, vol. 56, no. 10, pp. 4258-4265, Oct. 2009. Doi: 10.1109/tie.2009.2015754

[3] Y. Tan and S. Panda, "Optimized Wind Energy Harvesting System Using Resistance Emulator and Active Rectifier for Wireless Sensor Nodes," IEEE Transactions on Power Electronics, vol. 26, no. 99, p. 1, 2011. Doi 10.1109/tpel.2010.2056700

[4] S.E. Lyshevski, "High-power density miniscale power generation and energy harvesting systems" Energy Conversion and Management, Volume 52, Issue 1, January 2011, Pages 46-52. Doi 10.1016/j.enconman.2010.06.030.

[5] M. Piñuela, P. D. Mitcheson and S. Lucyszyn, "Ambient RF Energy Harvesting in Urban and Semi-Urban Environments", IEEE Trans. on Microwave Theory and Techniques, vol. 61, no. 7, pp. 2715-2726, 2013. Doi: 10.1109/tmtt.2013.2262687

[6] A. Dolgov, R. Zane, and Z. Popovic, "Power Management System for Online Low Power RF Energy Harvesting Optimization," IEEE Trans. on Circuits and Systems I: Regular Papers, vol. 57, no. 7, pp. 1802-1811, July 2010. Doi: 10.1109/tcsi.2009.2034891

[7] S. L. Brunton, C. W. Rowley, S. R. Kulkarni, and C. Clarkson, "Maximum Power Point Tracking for Photovoltaic Optimization Using Ripple-Based Extremum Seeking Control", IEEE Transactions on Power Electronics, vol. 25, no. 10, pp. 2531-2540, 2010. Doi: 10.1109/tpel.2010.2049747

[8] P. Di Marco, V. Stornelli, G. Ferri, L. Pantoli, A. Leoni, "Dual band harvester architecture for autonomous remote sensors", Sensors and Actuators A: Physical, Vol. 247, 15 August 2016, Pages 598-603, ISSN 0924-4247. Doi: 10.1016/j.sna.2016.06.040

[9] L. Pantoli, A. Leoni, V. Stornelli, G. Ferri, "An IC architecture for RF energy harvesting systems", Journal of Communication Software and Systems, vol. 13, p. 96-100, 2017. Doi: 10.24138/jcomss.v13i2.377

[10] V. C. Gungor and G. P. Hancke, "Industrial Wireless Sensor Networks: Challenges, Design Principles, and Technical Approaches", IEEE Transactions on Industrial Electronics, vol.56, no.10, pp. 4258-4265, 2009. Doi: 10.1109/tie.2009.2015754

[11] G. Saggio, F. Cavrini, C.A. Pinto, "Recognition of arm-and-hand visual signals by means of SVM to increase aircraft security", Studies in Computational Intelligence, 669, pp. 444-461, 2017. Doi: 10.1007/9783-319-48506-5_23

[12] Saggio, G., Lazzaro, A., Sbernini, L., Carrano, F. M., Passi, D., Corona, A., et al., "Objective surgical skill assessment: an initial experience by means of a sensory glove paving the way to open surgery simulation?", Journal of surgical education, 72(5), 910-917.

[13] Saggio, G., Bisegna, P., Latessa, G., \& Bocchetti, S. "Mechanical modeling of bend sensors exploited to measure human joint movements", IEEE International Symposium on World of Wireless, Mobile and Multimedia Networks \& Workshops, WoWMoM 2009, pp. 1-4. Doi: 10.1016/j.jsurg.2015.04.023

[14] G. Orengo, A. Lagati and G. Saggio, "Modeling Wearable Bend Sensor Behavior for Human Motion Capture", IEEE Sensors Journal, vol. 14, no. 
7, pp. 2307-2316, 2014. Doi: 10.1109/jsen.2014.2309997.

[15] S. Wise, W. Gardner, E. Sabelman, E. Valainis, Y. Wong, K. Glass, J. Drace, J. M. Rosen, "Evaluation of a fiber optic glove for semiautomated goniometric measurements", Journal of Rehabilitation Research and Development Vol. 27 No. 4, 1990 Pages 411-424. Doi: 10.1682/jrrd.1990.10.0411.

[16] G. Saggio, G. Orengo, A. Pallotti, V. Errico, M. Ricci, "Evaluation of an integrated sensory glove at decreasing joint flexion degree", IEEE MeMeA, 11-13 June 2018, Rome, Italy. Doi: 10.1109/MeMeA.2018.8438767

[17] A. Pallotti, M. Ricci, G.Orengo, G. Saggio, "Low Cost and Fast Development of 3D Printed Gloves for 10 Degrees of Freedom Gesture Recognition", Intern. Conf. on Biomedical Electronics and Devices (BIODEVICES), February 2019, Prague, Czech Republic

[18] R. Gentner, J. Classen, "Development and evaluation of a low-cost sensor glove for assessment of human finger movements in neurophysiological settings", J. Neurosci. Methods, 178, 138-147, 2009. Doi: 10.1016/j.jneumeth.2008.11.005

[19] L. K. Simone, N. Sundarrajan, X. Luoc, Y. Jia, D. G. Kamper, “A low cost instrumented glove for extended monitoring and functional hand assessment", Journal of Neuroscience Methods 160 (2007) 335-348. Doi: 10.1016/j.jneumeth.2006.09.021

[20] Lin J., Wu Y. and Huang T. S., "Modeling the constraints of human hand motion", IEEE Workshop on Human Motion, 121-126, 2000. Doi: 10.1109/HUMO.2000.897381

[21] G. Saggio, F. Riillo, L. Sbernini, L.R. Quitadamo, "Resistive flex sensors: A survey", Smart Materials and Structures, 25(1), 2015. Doi 10.1088/0964-1726/25/1/013001

[22] G. Saggio, M. De. Sanctis, E Giannini, "Long term measurement of human joint movements for health care and rehabilitation purposes", Wireless VITAE, Page 674-678, 2009

[23] G. Saggio, S. Bocchetti, C.A. Pinto, G. Orengo, F. Giannini, "A novel application method for wearable bend sensors", 2nd International Symposium on Applied Sciences in Biomedical and Communication Technologies, ISABEL 2009. Doi: doi: 10.1109/ISABEL.2009.5373625

[24] G. Saggio, M. Bizzarri,"Feasibility of teleoperations with multi-fingered robotic hand for safe extravehicular manipulations", Aerospace Science and Technology, vol. 39, pp. 666-674, 2014. Doi 10.1016/j.ast.2014.05.018

[25] G. Saggio, S. Bocchetti, G. Orengo, C. Pinto, "Electronic interface an signal conditioning circuitry for data glove systems useful as 3D HMI tools for disabled persons", $4^{\text {th }}$ International Conference on Health Informatics (HEALTHINF, part of BIOSTEC), Rome (Italy), 2011.

[26] G. Saggio, G. Orengo, "Flex sensor characterization against shape and curvature changes", Sensors \& Actuators A Physical., Vol. 273, pp. 221-231, 2018. Doi: 10.1016/j.sna.2018.02.035.

[27] Saggio G., Lazzaro A., Sbernini L., Carrano F. M., Passi D., Corona A., Panetta V., Gaspari A. L., Di Lorenzo N., "Sensory-Glove-Based Open Surgery Skill Evaluation", IEEE Transactions on Human-Machine Systems, Vol. 48, No. 2, April 2018. Doi: 10.1109/thms.2017.2776603

[28] P. D. Gleonec, J. Ardouin, M. Gautier and O. Berder, "Architecture exploration of multi-source energy harvester for IoT nodes," 2016 IEEE Online Conference on Green Communications (OnlineGreenComm), Piscataway, NJ, USA, 2016, pp. 27-32. Doi: 10.1109/OnlineGreenCom.2016.7805402

[29] J. J. Shi and X. D. Huang, "Electrostatic energy harvester based on chargetrapping nonvolatile memory structure," 2016 IEEE International Conference on Electron Devices and Solid-State Circuits (EDSSC), Hong Kong, Hong Kong, 2016, pp. 153-156. Doi: 10.1109/EDSSC.2016.7785232

[30] Z. Wang, W. Zhang, D. Jin, H. Xie, X. Lv, "A full-wave RF energy harvester based on new configurable diode connected MOSFETs," 2016 IEEE International Conference on Microwave and Millimeter Wave Technology (ICMMT), Beijing, 2016, pp. 117-119. Doi: 10.1109/ICMMT.2016.7761695.

[31] P. Broutas, H. Contopanagos, D. Tsoukalas, S. Chatzandroulis," A RF power harvester with integrated antenna capable of operating near ground planes", Sensors and Actuators A: Physical, Volume 186, October 2012, Pages 284-288, ISSN 0924-4247. Doi: 10.1016/j.sna.2012.05.040.

[32] M. Sun, D. Ranasinghe and S. F. Al-Sarawi, "RF energy harvester with peak power conversion efficiency tracking," 2016 IEEE Asia Pacific Conference on Circuits and Systems (APCCAS), Jeju, South Korea 2016, pp. 107-110. Doi: doi: 10.1109/APCCAS.2016.7803908.

[33] A. Leoni, V. Stornelli, G. Ferri, V. Errico, M. Ricci, A. Pallotti, G. Saggio, "A human body powered sensory glove system based on multisource energy harvester," 2018 14th Conference on Ph.D. Research in Microelectronics and Electronics (PRIME), Prague, 2018, pp. 113116. doi: 10.1109/PRIME.2018.8430362

[34] V. Stomelli, A. Leoni, G. Ferri, V. Errico, M. Ricci, A. Pallotti, G. Saggio, "A Multi-Source Energy Harvesting Sensory Glove Electronic Architecture," 2018 3rd International Conference on Smart and Sustainable Technologies (SpliTech), Split, 2018, pp. 1-4

[35] A. Leoni, L. Pantoli, V. Stornelli, G. Ferri, M. Russo and P. Solic, "90/900 MHz IC architecture for autonomous systems," 2017 2nd International Multidisciplinary Conference on Computer and Energy Science (SpliTech), Split, 2017, pp. 1-4.

[36] A. Leoni, L. Pantoli, V. Stornelli, G. Ferri, P. Solic and M. Russo, "A Combined 90/900 MHz IC Architecture for Smart Tag Application", Journal of Communications Software and Systems, vol. 14, no. 1, 2018 Doi: $10.24138 /$ jcomss.v14i1.451

[37] L. Pantoli, A. Leoni, V. Stornelli and G. Ferri, "Energy harvester for remote sensors systems", 2016 International Multidisciplinary Conference on Computer and Energy Science (SpliTech), 2016. Available: 10.1109/splitech.2016.7555933 [Accessed 14 January 2019].

[38] P. Di Marco, A. Leoni, L. Pantoli, V. Stornelli and G. Ferri, "Remote sensor networks with efficient energy harvesting architecture", 2016 12th Conference on Ph.D. Research in Microelectronics and Electronics (PRIME), 2016. Available: 10.1109/prime.2016.7519524 [Accessed 14 January 2019].

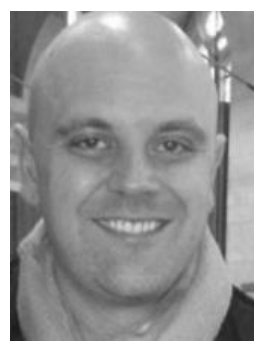

Vincenzo Stornelli was born in Avezzano, Italy. $\mathrm{He}$ received the "Laurea" degree (cum laude) in electronic engineering in 2004. In October 2004, he joined the Department of Electronic Engineering, University of L'Aquila, L'Aquila, Italy, where he is involved as Associate His research interests include several topics in computational electromagnetics, including microwave antenna analysis for outdoor ultrawideband applications. He serves as a reviewer for several international journals and Editor of the Journal of Circuits, Computers and Systems.

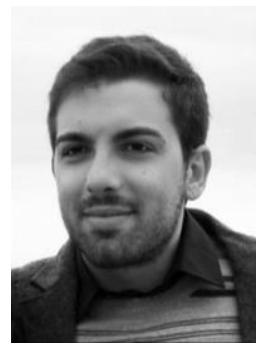

Alfiero Leoni was born in L'Aquila, Italy. He received the Bachelor "Laurea" degree in 2013 and master "Laurea" degree (cum laude) in electronic engineering in 2016. Several months before his master Laurea degree, he joined as an external collaborator the Department of Industrial and Electronic Engineering of the University of L'Aquila where he is enrolled as Ph.D. student, at the moment. His research activity mainly consists of the design of analog electronic and microelectronic circuits and systems for energy harvesting, industrial and microwave applications.

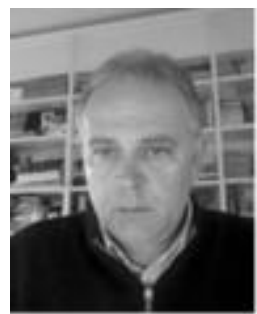

Giuseppe Ferri was born in L'Aquila, Italy. He received the "Laurea" degree (cum laude) in electronic engineering in 1988. In 1991, he joined the Department of Electronic Engineering, University of L'Aquila, L'Aquila, Italy, where he is actually a full professor of Electronics and Microelectronics at the University of L'Aquila, Italy. His research activity mainly concerns the design of analog electronic circuits for integrated sensor applications both in voltage and in currentmode. In this field of research, he is author or coauthor of 6 patents, 3 international books, one book chapter and more than 400 publications in international journals and conference proceedings. He is an IEEE senior member and Editor of Sensors and of Journal of Circuits, Computers and Systems. 


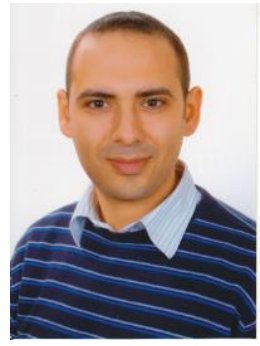

Vito Errico graduated in micro-electronic engineering at Polytechnic of Bari (Italy) in 2005 and received the $\mathrm{PhD}$ degree in materials and innovative technologies (ISUFI) from "Università degli Studi del Salento" (Italy) in 2008. In the same year, he was appointed to post-doctoral researcher position at the University of Birmingham, United Kingdom. From 2013 he hold research fellowship positions at University of Rome "Tor Vergata", Italy.

Currently, his research interests involve sensors, biotechnologies, human-machine systems and motion capture.

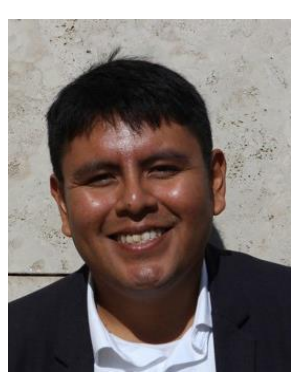

applications.

Antonio Pallotti received his M.Sc. in Medical Engineering from University of Rome Tor Vergata. Now he is a Ph.D. candidate in Electronic Engineering at the University of Rome Tor Vergata, Research Fellow at the Technoscience and Adjunct Lecturer in Bioengineering at the University of Rome San Raffaele. During his PhD he was the inventor and developer of the 3D printed glove, the developer of lycra glove. He is specialized in wearable sensors developement, machine learning and telemonitoring for health

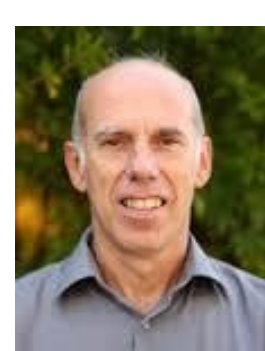

Giancarlo Orengo received his degree in Electronics Engineering from the University of Rome, Italy, in 1989. From 2004 he is associate professor of electronics. He worked on MMIC's design, characterization and modeling with III-IV devices. He was envolved in design and realization of analog/digital IC front-end electronics in LHC (Large Hadron Collyder) at the CERN of Geneva. In the last years his scientific activity has been extended to the integration of different sensing technologies for human posture recognition and motion capture.

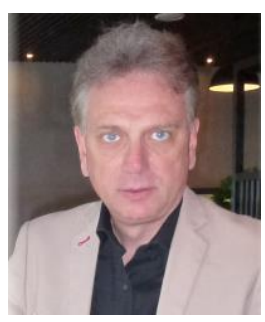

Giovanni Saggio (GS) received the degree in Electronics Engineering and the $\mathrm{PhD}$ in MicroElectronics and Telecommunication Engineering at the University of Rome "Tor Vergata" (Italy). GS is Researcher and Aggregate Professor at the University of Rome "Tor Vergata", holds chairs about Electronics at the Engineering Faculty and at the Medical Faculty. GS authored or co-authored about 200 scientific publications, eight patents, several book chapters and is unique author of four books about electronics. GS's research interests involve sensors, biotechnlogies, human-machine systems, motion capture and machine learning. GS co-founded the Captiks enterprise (www.captiks.com) involved in systems to measure and analyze human kinematics, and the Seeti enterprise (www.seeti.it) involved in virtual and augmented reality systems. 